\title{
El fenómeno de la deserción escolar en un contexto local: estudio de la política municipal
}

Fernelly Varón-Martínez ${ }^{*}$

\footnotetext{
Especialista en Gerencia de Instituciones Educativas. Rector, Colegio Akademos, Cali, Colombia.

Correo electrónico:

fernellyvar@hotmail.com
}

Recibido: 25 de febrero del 2017

Aprobado: 15 de abril del 2017

Cómo citar este artículo: Fernelly VarónMartínez. El fenómeno de la deserción escolar en un contexto local: estudio de la política municipal. DIXI 26. Mayo 2017. Pág. 85. doi: http://dx.doi.org/10.16925/di.v19i26.1953

\section{Resumen}

Propósito: este reporte analiza el problema de la deserción estudiantil en la Institución Educativa Siete de Agosto, de Cali, por medio del estudio de los factores internos y externos y el análisis del desarrollo de los procesos educativos como causantes del retiro escolar. Se sugieren también estrategias encaminadas a disminuir el riesgo de desertar. Descripción: para esto se realiza un estudio exploratorio, y se utiliza el análisis cuantitativo y cualitativo de la comunidad educativa relacionada con este fenómeno educativo, con un especial interés por el ser. Punto de vista: particularmente, se identifica una tendencia de retiro escolar con diversidad de variables en un periodo de tiempo específico dentro del marco temporal seleccionado. Conclusiones: los resultados de esta investigación señalan que los niños y los jóvenes se retiran de la institución por movilidad o desplazamiento intraurbano, problemas de convivencia, fallas en la función administrativa de la institución y otras problemáticas socio-culturales.

Palabras clave: deserción escolar, estudio exploratorio, retención, variables. 


\title{
The Phenomenon of School Dropout in a Local Context: A Study of Municipal Policy
}

\begin{abstract}
Purpose: This report analyzes the problem of student dropout at the educational institution Siete de Agosto in Cali by studying internal and external factors and the analysis of the development of educational processes as causes of school dropout. Strategies to reduce the risk of dropout are also suggested. Description: For this, an exploratory study is conducted, and the quantitative and qualitative analysis of the educational community related to this educational phenomenon is used, with a special interest in the individual. Point of view: Particularly, a school dropout trend is identified with diverse variables in a specific period of time within the selected timeframe. Conclusions: The results of this research indicate that children and young people leave the institution due to intra-urban mobility or transportation, problems of coexistence, failures in the institution's administrative function, and other socio-cultural problems.
\end{abstract}

Keywords: school dropout, exploratory study, retention, variables.

\section{O fenômeno da deserção escolar num contexto local: estudo da política municipal}

\section{Resumo}

Propósito: este relatório analisa o problema da deserção estudantil na Instituição Educativa Siete de Agosto, Cali, Colômbia, por meio do estudo dos fatores internos e externos, bem como da análise do desenvolvimento dos processos educativos como causantes do abandono escolar. Sugerem-se também estratégias encaminhadas a diminuir o risco de deserção. Descrição: para isso, realiza-se um estudo exploratório e utiliza-se a análise quantitativa e qualitativa da comunidade educativa relacionada com esse fenômeno educativo, com especial interesse por ele. Ponto de vista: em particular, identifica-se uma tendência de abandono escolar com diversidade de variáveis num período específico. Conclusões: os resultados desta pesquisa indicam que as crianças e os jovens abandonam a instituição por mobilidade ou deslocamento intraurbano, problemas de convivência, falhas na função administrativa da instituição e outras problemáticas socioculturais.

Palavras-chave: deserção escolar, estudo exploratório, retenção, variáveis. 


\section{INTRODUCCIÓN ${ }^{1}$}

El fenómeno de la deserción, considerado como el abandono del sistema educativo por parte de los estudiantes, es una problemática compleja que afecta por igual a todo el sistema educativo colombiano, y en particular a las instituciones del sector público, como es el caso de la Institución Educativa Siete de Agosto de Cali, Colombia.

Los diferentes problemas sociales, económicos, familiares e individuales parecen determinar los comportamientos del estudiante que deserta del sistema. Sin embargo, se encuentra otra dimensión importante del fenómeno, la cual tiene su arraigo en el interior mismo de la escuela; esta se relaciona con la responsabilidad social, los mecanismos de evaluación $y$, en general, la calidad del servicio que se ofrece.

La preocupación por el tema de la deserción escolar ocupa a múltiples sectores. Por ejemplo, la CEPAL realizó, en el 2012, ${ }^{2}$ un completo informe sobre deserción escolar en América Latina. En el contexto nacional, es significativo el informe de la Contraloría General de la República. ${ }^{3}$ También existen amplios estudios que abordan y contextualizan el tema desde diversas disciplinas de la ciencias sociales, como es el caso del trabajo teórico de Vincent Tinto, ${ }^{4}$ autor que resalta la deserción como fenómeno psicológico, ya que en este se ven comprometidos asuntos y decisiones individuales. El autor destaca a su vez el aspecto societal referido a los éxitos o fracasos sociales, y sostiene que la deserción se encuentra ligada a problemas de orden económico y organizacionales.

El grupo investigativo realiza el estudio exploratorio sobre la deserción en la Institución Educativa Siete de Agosto durante los últimos tres periodos escolares, en razón a los aspectos que se presentan a continuación.

El proyecto es pertinente porque la comunidad, en general, está en búsqueda de alcanzar la calidad y la responsabilidad social de la escuela frente a las variables motivantes de la deserción. Es viable porque requiere de la implementación de procesos de autogestión, en los que la calidad y la satisfacción del

1. Estudio exploratorio de la deserción escolar en la Institución Educativa Siete de Agosto de Cali, durante el periodo 2005-2016.

2. Cf. Comisión Económica para América Latina y el Caribecepal. Deserción Escolar en América latina. Informe. CEPAL. (2012).

3. Cf. Contraloría General de la República y Dirección de Estudios Sectoriales. LA DESERCIÓN ESCOLAR EN LA EDUCACIÓN BÁSICA Y MEDIA. (2004).

4. Cf. Vincent Tinto. Definir la deserción: una cuestión de perspectiva. Revista de Educación Superior 71. 1989. Pág. 1-43. cliente en el ofrecimiento del servicio propendan a la retención de los jóvenes dentro del sistema educativo. Por último, es útil porque existe la necesidad de crear procesos sociales incluyentes desde la escuela, en busca de la disminución de los niveles de deserción que se presentan en la Institución Educativa Siete de Agosto, durante los tres últimos periodos lectivos en los cuales las cifras de cobertura y prestación del servicio han disminuido significativamente.

\section{Antecedentes}

Con relación a la problemática de la deserción escolar, se han realizado diversos estudios que determinan como causales los factores de tipo individual, socioeconómicos y académicos, los cuales se enmarcan dentro de espacios locales, nacionales e internacionales.

\section{CONTEXTO INTERNACIONAL}

\section{A. Informe CEPAL sobre deserción escolar en América Latina 2012}

Según el informe sobre la deserción escolar en América Latina, realizado por la CEPAL en 18 países de América Latina, hacia el 2012, cerca de 15 millones de jóvenes de entre 15 y 19 años de edad, de un total de 49,4 millones, habían abandonado la escuela antes de completar 12 años de estudio. Alrededor del $70 \%$ de ellos lo habían hecho temprano, antes de completar la educación primaria o una vez terminada. A pesar de que la brecha ha disminuido en la última década, las diferencias entre el contexto rural y el urbano son importantes: al inicio del milenio, la tasa total de deserción en zonas rurales (48 \%) casi duplicaba a la tasa urbana (26\%). Los datos provienen de la última publicación de Panorama Social de América Latina (CEPAL), en la que se indica que abordar este problema es uno de los principales desafíos para alcanzar las metas de desarrollo social planteadas por las Naciones Unidas para el 2015. Se enfatiza la necesidad de que los países de la región destinen mayores recursos a políticas y programas dirigidos a evitar que los niños interrumpan sus estudios antes de terminar el ciclo básico y a procurar una disminución significativa de la deserción en el ciclo medio. ${ }^{5}$

\footnotetext{
5. Véase Comisión Económica para América Latina y el CaribeCEPAL, supra, nota 2.
} 
Puntualiza la CEPAL la problemática latinoamericana de deserción con cifras en las que se visualiza el abandono escolar antes de completar el ciclo secundario en 18 países de América Latina, basadas en datos de encuestas de hogares. A fin de analizar el abandono escolar durante los ciclos primario y secundario, se elaboró una clasificación que describe la situación escolar de los adolescentes entre 15 y 19 años. Los datos obtenidos indican grandes diferencias entre países, así como entre los contextos urbano y rural.

\section{B. Datos generales}

El mismo informe señala que, en promedio, cerca del 37 \% (unos 15 millones) de los adolescentes latinoamericanos entre 15 y 19 años de edad abandonan la escuela a lo largo del ciclo escolar, y casi la mitad de los que desertan lo hacen muy pronto, antes de completar la educación primaria. Sin embargo, en varios países, la mayor parte de la deserción se produce una vez completado ese ciclo $y$, frecuentemente, durante el transcurso del primer año de la enseñanza media. A lo anterior hay que agregar 1,4 millones de niños y niñas que nunca asistieron a la escuela o la abandonaron antes de completar el primer año básico. ${ }^{6}$

\section{Diferencias según nivel educativo del país}

En aquellos países que han logrado niveles educativos relativamente más altos, la tasa global de deserción en las zonas urbanas fluctúa entre $16 \%$ y $25 \%$; en otro grupo, el abandono escolar oscila en torno a un promedio de $37 \%$, mientras que, en un reducido número de países, con un nivel más bajo de cobertura de la educación primaria, la deserción afecta a entre el $40 \%$ y el $50 \%$ de los adolescentes. Así, en las zonas urbanas de Argentina, Chile y Panamá, la tasa global de deserción durante los ciclos primario y secundario afecta a uno de cada cinco adolescentes, elevándose a uno de cada tres en las zonas urbanas de El Salvador, Guatemala y Nicaragua. En las zonas rurales, en Brasil, Colombia y Perú, dos de cada cinco adolescentes entre 15 y 19 años abandonan la escuela antes de completar la secundaria, mientras que en Bolivia, Honduras y México lo hacen dos de cada tres, y en ambos grupos de países alrededor del $80 \%$ de la deserción escolar se concentra durante o al finalizar la educación primaria.

6. Id. Págs. 10-80.

\section{Diferencias por sexo}

En las zonas urbanas, las niñas abandonan la escuela con menor frecuencia que los niños; progresan a lo largo del ciclo escolar con menor repetición, y en una mayor proporción las jóvenes con relación a los varones entre 15 y 19 años de edad regresan de la educación secundaria sin retraso. En las zonas rurales, en cambio, las mujeres tienden a dejar la escuela más temprano que los hombres, particularmente durante los primeros años de la primaria, y en algunos países (Bolivia, Guatemala, Paraguay, Perú y República Dominicana), un porcentaje mayor de niñas que de niños no ingresan al sistema o lo abandonan sin completar el primer grado.

\section{E. Deserción temprana}

¿En qué etapas del ciclo educacional tiende a concentrarse actualmente la deserción escolar en los países? La situación regional es la siguiente.

\section{En las zonas urbanas}

- En Bolivia, Brasil, El Salvador, Guatemala, Nicaragua, República Dominicana y Venezuela, entre el $40 \%$ y más del $70 \%$ de los niños y niñas dejan de asistir a la escuela antes de completar el ciclo primario de los respectivos países (remítase a la Gráfica 1 del informe de la Contraloría General de la República).

- En Chile, Colombia, México, Panamá, Perú y Uruguay, entre el $50 \%$ y $60 \%$ del abandono escolar se produce en el transcurso de la secundaria y, con excepción de Chile, en todos ellos la deserción se concentra más en el comienzo que en el final del ciclo.

- En Argentina, Costa Rica, Ecuador, Honduras y Paraguay, más de la mitad y hasta el $60 \%$ de los que abandonan la escuela lo hacen al finalizar el ciclo primario. (Si bien solo cinco países concentran la mayor parte de la deserción al finalizar la primaria, el abandono escolar en esta etapa es importante en las zonas urbanas, ya que en otros nueve países el porcentaje de retiro de la educación formal, completado dicho ciclo, fluctúa entre el $23 \%$ y el $35 \%$ ).

\section{En las zonas rurales}

- La deserción ocurre casi totalmente o con mucha mayor frecuencia durante el ciclo primario, y en 
algunos de los países un porcentaje muy bajo de los niños logran completar dicho ciclo (Brasil, El Salvador, Guatemala, Nicaragua, República Dominicana y Venezuela).

- Solo en Chile, Colombia, México, Panamá y Perú - países en los que una fracción relativamente más alta de los niños de zonas rurales logran acceder a la educación secundaria-, entre un $20 \%$ y un $40 \%$ del total abandona la escuela en el transcurso de ese ciclo. Los más elevados costos sociales y privados (en términos de pérdidas de ingresos futuros en el mercado de trabajo) que derivan de una deserción escolar temprana - es decir, aquella ocurrida antes de completar el número de años que contempla el ciclo primario de cada país-, señalan la necesidad de concentrar los esfuerzos de retención escolar en los primeros años de ese ciclo (remítase a las Gráficas 2 y 3 del informe de la Contraloría General de la República).

\section{Contexto Nacional}

\section{A. Informe Contraloría General de la República}

Según el informe de la Contraloría General de la República, ${ }^{7}$ las cifras del 2004 muestran que solo el $47 \%$ de los estudiantes que inician el ciclo completo de educación, en Colombia, lo termina. El mismo documento agrega que de cada cien matriculados, siete, en promedio, abandonan cada año la escuela. Solo en ese año abandonaron el servicio educativo 760000 estudiantes, lo cual implicó que el Estado y la sociedad perdieran, por causa de este fenómeno, más de cop 680000 millones invertidos. En términos territoriales, amplia el informe, las regiones más afectadas son los antes denominados "territorios nacionales", así como los departamentos más pobres del país. En Guainía, Putumayo, Guaviare y Caquetá, la tasa de deserción escolar es de más del doble del promedio nacional. Además, destaca el texto mencionado que la deserción escolar en el Eje Cafetero supera en más del $40 \%$ el índice nacional. Asimismo, regiones favorecidas por la asignación de altos recursos de regalías, tales como Arauca, Casanare y Meta, tienen altos índices de desertores escolares. El informe concluye en esta parte que estos indicadores ameritan un estudio detallado y una pronta solución.

Véase Contraloría General de la República-Dirección de Estudios Sectoriales, supra, nota 3.
Para el estudio del fenómeno de la deserción escolar, se han agrupado los factores que la determinan en tres grandes temas, aunque, claro está, están interrelacionados. El primero se refiere a la institucionalidad en la prestación del servicio educativo; el segundo tiene que ver con los factores pedagógicos y propios de la docencia; y el tercero se relaciona con el entorno social y económico.

\section{B. Factores institucionales}

La educación - según el "Informe de la Misión de los Sabios. Colombia al filo de las oportunidades", en su apartado "La base" - ${ }^{8}$ así como la ciencia y el desarrollo, se han percibido en el contexto colombiano y a lo largo de su historia como un gasto, no como una inversión a corto, mediano o largo plazo. En la medida en que los individuos tengan la posibilidad de acceder a espacios educativos, se generarán conductas adecuadas para la convivencia, el aprovechamiento de los saberes locales y universales, y la interrelación con el medio ambiente. Con la educación se tiene la posibilidad de acceder a saberes que han determinado nuestro pasado, saberes que inciden en nuestro presente y que con toda seguridad nos proyectarán a un mejor futuro. La formación académica e integral de los individuos, integralidad que, para el caso colombiano se encuentra formalizada en la ley general de educación, se ha consolidado como la mejor herramienta del desarrollo progresista de la humanidad.

El panorama de la educación, según el documento Colombia al filo de las oportunidades, visiona los procesos formativos de los jóvenes en la escuela como factores determinantes frente al reto de la globalización, en el cual el conocimiento juega un papel importante en el desarrollo de los pueblos latinoamericanos, a partir del aprovechamiento de la ciencia y la tecnología. Los gobiernos deben generar políticas serias frente a la calidad y la cobertura educativa. Estas políticas deben iniciarse desde la familia y luego en la escuela. Desde el hogar, se debe empezar a formar los hombres de bien, capaces de convivir en medio de valores tales como la tolerancia, el respeto, la honradez y el civismo, entre otros; se debe mirar a la familia como actor fundamental en los procesos educativos. En la escuela se empiezan a trazar los objetivos que llevan a los individuos colombianos a

8. Véase Eduardo Aldana-Valdés et al. Misión, Ciencia, Educación y Desarrollo. Tomo 1. Informe DE LA Misión DE LOS SABIOS. COLOMBIA: AL FILO DE LA OPORTUNIDAD. Colciencias, TM Editores. 
mejorar sus condiciones de vida y a ejecutar un proyecto de vida digno.

En relación con las telecomunicaciones y los medios masivos de comunicación, estos se deben concebir como herramientas positivas de intercambio cultural de saberes e información, y no como elementos alienantes y superfluos que propendan a la deshumanización y la abstracción de quienes llegan a la escuela y no asumen con criterios serios sus procesos de formación.

Debe mirarse también hacia la comunidad y verla como un actor principal de los procesos internos y externos de las instituciones. La elaboración de currículos integrados, pertinentes y producto de experiencias investigativas en el contexto, serán un aporte valioso en la búsqueda de la calidad.

El Gobierno debe generar las condiciones y los mecanismos para que la calidad de la educación colombiana mejore, por lo tanto, se hace necesario un incremento a la inversión en educación, lo cual a través de los años se ve reducido, tal como lo plantean las recientes reformas a la ley de transferencias. ${ }^{9}$ La creación de normas, como la denominada "promoción automática" de hace algunos años, o el actual Decreto 0230 del 2002, por el cual se dictan normas demasiados flexibles sobre promoción de estudiantes, encaminan al sistema educativo a elevados índices de cobertura, y atacan con esto unas de las variables de deserción como es la reprobación, pero descuidan el nivel de exigencia académico y disminuyen con esto la calidad y la competitividad del estudiante que se forma.

Se hace necesaria la búsqueda de una identidad nacional en la que se valoren y se integren lo pluricultural y lo étnico del país. Lograr la participación de todos los colombianos en la elaboración de un sistema educativo pertinente con su realidad nacional, alimentará, en gran medida, una identidad local que, lamentablemente, se ha perdido por la incorporación de modelos educativos ajenos a su realidad y contexto.

Se puede competir y enfrentar la globalización con criterios propios, implementados desde la escuela, donde el conocimiento impartido se vea sustentado por políticas sociales y económicas que

\footnotetext{
9. Véase Acto legislativo 01 del 2001. Por medio del cual se modifican algunos artículos de la Constitución Política. Agosto 1 del 2001. Do 44506; véase también Ley 715 del 2001. Por la cual se dictan normas orgánicas en materia de recursos y competencias de conformidad con los artículos 151, 288, 356 y 357 (Acto Legislativo 01 de 2001) de la Constitución Política y se dictan otras disposiciones para organizar la prestación de los servicios de educación y salud, entre otros. Disponible en http://www.mineducacion.gov. co/1621/articles-86098_archivo_pdf.pdf
}

complementan la función transformadora de la educación desde las instituciones educativas y fuera de ellas.

Dentro de los presupuestos y determinantes de las reformas educativas, se evidencia que países en avanzado estado de desarrollo tales como los Estados Unidos, Francia, Japón e Inglaterra han realizado reformas educativas propias de sus realidades socio-económicas. Países latinoamericanos como Chile, Argentina y, ahora, Colombia implementan las políticas exigentes de la corriente neoliberal y de la globalización, en las que las reformas propuestas por estas tendencias - de manera lamentable para estas naciones - tocan básicamente aspectos administrativos y presupuestales, y muy superficialmente propuestas serias frente a lo curricular o lo pedagógico. Los recortes a la inversión en educación y salud, para citar solo dos aspectos básicos, corresponden a exigencias del Fondo Monetario Internacional (FMI) y del Banco Interamericano de Desarrollo (BID), con el fin de ampliar la capacidad de endeudamiento de los países, y no como respuesta a políticas serias sobre la optimización de los recursos públicos y la eficiencia de las entidades oficiales.

La intención de la Ley 115 de 1994, en su planteamiento sobre la libertad de las instituciones y su autonomía para la elaboración del proyecto educativo institucional (PEI), no logró materializarse:

Currículo es el conjunto de criterios, planes de estudio, programas, metodologías, y procesos que contribuyen a la formación integral y a la construcción de la identidad cultural nacional, regional y local, incluyendo también los recursos (...) y llevar a cabo el PEI. ${ }^{10}$

El diseño curricular de las instituciones y su pertinencia con entornos locales, regionales y nacionales no se realizó y, después de los plazos considerados oportunos por el Ministerio de Educación Nacional (MEN), se crearon los estándares de calidad y las competencias específicas, lo cual significó una vez más la centralización y unificación del sistema educativo, tal como se había planteado en la reforma curricular de 1978:

Establecimiento de un currículo nacional por áreas. Materiales basados en el currículo. Control de textos y materiales educativos para estudiantes. Capacitación docente orientada por el currículo preestablecido.

10. Véase Ley 115 de 1994. Por la cual se expide la ley general de educación. Febrero 8 de 1994. Art. 49. Disponible en http://www. mineducacion.gov.co/1621/articles-85906_archivo_pdf.pdf 
Currículo (Art 4, Decreto 1419) Centrado en el estudiante para su desarrollo integral. Equilibrio entre teoría y práctica. Sistema orientado a la formación personal y a la interpretación social. Promover el estudio de problemas nacionales e internacionales. ${ }^{11}$

Se hace necesario entonces la creación de currículos pertinentes que se conviertan en elementos llamativos y atractivos para los jóvenes y la comunidad educativa en general, al momento de tomar decisiones frente a la escuela y la deserción.

Por otra parte, se hace necesaria la unificación de criterios frente a los diferentes niveles de educación. Desde la formulación de la Constitución Nacional, ${ }^{12}$ se ve un compromiso expreso del Gobierno por cumplir con la educación preescolar y básica (primaria y secundaria), pero no se asume la responsabilidad sobre la media (grados $10^{\circ}$ y $\left.11^{\circ}\right)$,lo que deja esto como rueda suelta que con el transcurrir de los años será asumida por el sector privado, previa articulación con lo público. Esta problemática se puede analizar de la siguiente manera.

La problemática educativa por niveles pone en evidencia la fragmentación del proceso formativo de los niños y los jóvenes, a causa de la falta de unidad en los criterios de cobertura, currículo y evaluación.

En el informe de la Contraloría General de la República (remítase a la Gráfica 4 de ese informe), ${ }^{13} \mathrm{se}$ muestra cómo el marco regulatorio para la educación preescolar, básica y media en Colombia ha generado una institucionalidad que impacta sobre el fenómeno de la deserción escolar. Por una parte, la búsqueda de la eficiencia fiscal y de asignación establecida en la Ley 715 ha llevado a incrementos significativos en la cobertura de matrícula en el sector oficial, lo cual contrasta con una dramática disminución de esta del sector no oficial, tanto a nivel agregado, como en la mayoría de las entidades territoriales del país.

El Ministerio de Educación Nacional ${ }^{14}$ complementa el anterior cuadro estadístico al explicar cómo, en el 2007, más de 11 millones de estudiantes

\footnotetext{
11. Véase Ministerio de Educación Nacional. Colombia REFORMA CURRICULAR. (1978).

12. Véase Constitución Política de Colombia [Const]. Título II de los Derechos, garantías y deberes, Capítulo I de los Derechos Fundamentales. Julio 7 de 1991 (Colombia).

13. Véase Contraloría General de la República-Dirección de Estudios Sectoriales, supra, nota 3.

14. Véase Ministerio de Educación Nacional. Sistema Nacional de Indicadores Educativos para los Niveles de Preescolar, Básica y Media en Colombia. Disponible en: /www.mineducacion.gov.co/1621/articles-329021_archivo_pdf_indicadores_educativos_enero_2014.pdf
}

asistieron a la educación básica primaria, secundaria y media. La cobertura bruta de educación básica llegó al 100,87 \%. Según la información reportada por las secretarías de educación, 9170199 estudiantes fueron atendidos en establecimientos oficiales y 1873646 por establecimientos no oficiales (remítase a la Tabla 1 del informe de la Contraloría General de la República).

Los 24649 establecimientos educativos se encuentran distribuidos en 14110 oficiales y 10539 no oficiales; la integración de sedes ha buscado ofrecer la continuidad a los estudiantes, al pasar de la primaria a la secundaria, y de esta a la media.

Se observa cómo, a partir del 2003, se empieza a presentar una disminución en la matrícula de las instituciones no oficiales con relación a las oficiales, registrándose una estabilización en ese mismo año y en el 2004; luego se aprecia una caída significativa pero estabilizada en los tres años siguientes. Se puede concluir que, a partir del 2012, se observa un incremento en el total del número de matriculados, debido, principalmente, al comportamiento del sector oficial, lo que contrasta con la notoria disminución en el sector no oficial, variación que se explica por la implementación de los planes de eficiencia en el servicio educativo a partir de la vigencia de la Ley 715 de 2001.

Cuando se manifiesta una reducción en la matrícula de las instituciones no oficiales, se genera al mismo tiempo una exigencia para el Gobierno Nacional en términos de inversión, porque se debe asumir la formación de aquellos jóvenes que el sector privado no está en condiciones de atender por diversos factores, entre ellos la deserción, la cual a su vez es motivada por variables de diversos tipos. En fin, el Estado debe garantizar la cobertura educativa para todos los jóvenes y los niños del país.

El informe de la Contraloría hace referencia al Decreto 2833 de 2004, por el cual se establece que la asignación de los recursos para la ampliación de cobertura (población por atender), se hará, a partir del 2005, con base en la diferencia de población matriculada en la vigencia actual, menos la matriculada en la vigencia anterior, restándole la disminución de la matrícula en el sector no oficial. En otras palabras, el traslado de matrícula del sector no oficial al oficial, implica que la incapacidad de retención del estudiante en el sector privado afecta los recursos que las entidades territoriales deben destinar a aumento de cobertura.

Se notan progresos significativos en el esfuerzo del gobierno por brindar cobertura educativa al asignar nuevos cupos en las instituciones educativas 
oficiales, o bien al realizar acuerdos de ofrecimiento del servicio con instituciones privadas, pero no se han generado estrategias serias frente a uno de los fenómenos que atenta contra esta sana intención de ofrecer educación a la gran mayoría de niños y jóvenes en nuestro país, como lo es la deserción. A lo anterior se puede agregar la problemática de corrupción que presentan quienes manejan los presupuestos locales de educación, quienes estuvieron recientemente involucrados en el robo de los dineros asignados a la articulación de la cobertura con el sector privado.

La Contraloría General explica en su informe el fenómeno de la deserción escolar al señalar cómo la población total matriculada en educación básica y media en el 2004 fue de 10524547 estudiantes, con una tasa de deserción promedio del 7,05 \% anual (remítase a la Tabla 2 del informe de la Contraloría General de la República), y establece que solo terminaría el ciclo completo de educación el 47,31 \% de los estudiantes que lo iniciaron, es decir, de cada 100 niños que ingresan al sistema escolar, solo 53 culminaran el ciclo. Así, las cifras muestran cómo en el 2004 abandonarían el servicio educativo 758956 estudiantes que, a un valor promedio por estudiante de COP 896292 (asignación por alumno de los Recursos del Sistema General de Participaciones para el 2004), equivaldrían a COP 680376 millones de pesos que el Estado y la sociedad estarían perdiendo por este fenómeno.

En este sentido, la Ley 715 del 2001, en el Capítulo IV "Distribución de recursos del sector educativo", en su Artículo 16, señala:

Artículo 16. Criterios de distribución. La participación para educación del Sistema General de Participaciones será distribuida por municipios y distritos atendiendo los criterios que se señalan a continuación. En el caso de municipios no certificados los recursos serán administrados por el respectivo Departamento.

Población atendida

Anualmente se determinará la asignación por alumno, de acuerdo con las diferentes tipologías educativas que definirá la Nación, atendiendo, los niveles educativos (preescolar, básica y media en sus diferentes modalidades) y las zonas urbana y rural, para todo el territorio nacional.

Se entiende por tipología un conjunto de variables que caracterizan la prestación del servicio educativo en los niveles de preescolar, básica y media, de acuerdo con metodologías diferenciadas por zona rural y urbana. Dentro de una misma tipología la asignación será la misma para todos los estudiantes del país.
Las tipologías que se apliquen a los departamentos creados por la Constitución de 1991, deberán reconocer sus especiales condiciones para la prestación del servicio público de educación, incluida la dispersión poblacional.

La asignación por alumno en condiciones de equidad y eficiencia según niveles educativos (preescolar, básica y media en sus diferentes modalidades) y zona (urbana y rural) del sector educativo financiado con recursos públicos, está conformado, como mínimo por: los costos del personal docente y administrativo requerido en las instituciones educativas incluidos los prestacionales, los recursos destinados a calidad de la educación que corresponden principalmente a dotaciones escolares, mantenimiento y adecuación de infraestructura, cuota de administración departamental, interventoría y sistemas de información.

La Nación definirá la metodología para el cálculo de la asignación por alumno y anualmente fijará su valor atendiendo las diferentes tipologías, sujetándose a la disponibilidad de los recursos del Sistema General de Participaciones.

La asignación por alumno se multiplicará por la población atendida con recursos del Sistema General de Participaciones en cada municipio y distrito. El resultado de dicha operación se denominará participación por población atendida, y constituye la primera base para el giro de recursos del Sistema General de Participaciones.

La población atendida será la población efectivamente matriculada en el año anterior, financiada con recursos del Sistema General de Participaciones.

Cuando la Nación constate que, debido a deficiencias de la información, una entidad territorial recibió más recursos de los que le correspondería de conformidad con la fórmula establecida en el presente artículo, su participación deberá reducirse hasta el monto que efectivamente le corresponda. Cuando esta circunstancia se presente, los recursos girados en exceso se deducirán de la asignación del año siguiente.

Después de determinar la participación por población atendida, el conPEs anualmente, previo análisis técnico, distribuirá el saldo de los recursos disponibles atendiendo alguno o algunos de los siguientes criterios.

Población por atender en condiciones de eficiencia A cada distrito o municipio se le podrá distribuir una suma residual que se calculará así: se toma un porcentaje del número de niños en edad de estudiar que no están siendo atendidos por instituciones oficiales y no estatales, y se multiplica por la asignación de niño por atender que se determine, dándoles prioridad 
a las entidades territoriales con menor cobertura o donde sea menor la oferta oficial, en condiciones de eficiencia.

El CONPEs determinará cada año el porcentaje de la población por atender que se propone ingrese al sistema educativo financiado con los recursos disponibles del Sistema General de Participaciones durante la siguiente vigencia fiscal.

La asignación para cada niño por atender se calculará como un porcentaje de la asignación por niño atendido y será fijado anualmente por la Nación.

Cuando la matrícula en educación en una entidad territorial sea del 100\% de la población objetivo, ésta no tendrá derecho a recibir recursos adicionales por concepto de población por atender en condiciones de eficiencia. Igualmente, cuando la suma de los niños matriculados, más el resultado de la multiplicación del factor de población por atender que determine el CONPES por la población atendida, sea superior a la población objetivo (población en edad escolar), sólo se podrá transferir recursos para financiar hasta la población objetivo. ${ }^{15}$

En el artículo 2 de la misma ley, se hace referencia a la base de cálculo del sistema general de participaciones de la siguiente manera:

Los valores que sirven de base para establecer el Sistema General de Participaciones en 2012 corresponderán a los señalados en el parágrafo $1^{\circ}$ del artículo 357 de la Constitución Política, sin que en ningún caso el monto sea inferior a diez punto novecientos sesenta y dos (10.962) billones de pesos de 2001, y su crecimiento será el señalado en el mismo artículo. ${ }^{16}$

Se podría pensar que estos recursos son significativos, pero no cubren en gran parte las necesidades del sistema educativo, porque en su gran mayoría estos porcentajes son destinados a cubrir gastos de funcionamiento como las nóminas del personal docente y administrativo. Por lo tanto, el nivel de inversión es muy limitado y queda supeditado a lo que se pueda generar con el apoyo de otros decretos de ley como la Ley 21, de modo que la lentitud se hace evidente en la pobre y tardía ejecución de proyectos encaminados a mejorar aspectos de planta física y dotación de instituciones educativas delos niveles de básica y media.

15. Véase Ley 715 del 2001, supra, nota 9.

16. Id.

\section{Infraestructura de las instituciones educativas}

El informe de la Contraloría define la infraestructura de las instituciones educativas como los insumos educativos que contribuyen a la adquisición de habilidades de aprendizaje y que determina los logros del proceso educativo.

El documento refiere que dada la importancia que tiene la infraestructura en el desarrollo pedagógico institucional, es preciso procurar un entorno que brinde herramientas de desarrollo y formación integral, mediante bibliotecas, escenarios culturales y deportivos, materiales didácticos y herramientas tecnológicas para el manejo de la información. Sin embargo, todo esto requiere de mayores niveles de inversión, es decir, de gasto en educación, o por lo menos de mayor eficiencia y eficacia en el uso de los recursos existentes. Por ejemplo, los recursos provenientes de la Ley 21 de 1982 que se pueden usar en proyectos de infraestructura y dotación en instituciones de educación media, han presentado bajos niveles de ejecución. El informe concluye que, de hecho, de los proyectos presupuestados para el 2012, tan solo se ha finalizado el $10 \%$, y de los proyectos del 2004 y el 2005, no se ha culminado ninguno.

\section{Factores endógenos}

Los factores endógenos se analizan en el informe de la Contraloría a partir de dos aspectos: la función de los maestros y los procesos de evaluación en la escuela.

\section{Función motivadora del maestro}

Sobre la función motivadora del maestro, el documento de la Contraloría General de la República señala que es él quien asume la responsabilidad de ser transmisor y creador de valores culturales, consolidador del lenguaje y constructor de relaciones y redes sociales. En relación con la deserción escolar, el papel del docente es fundamental, toda vez que puede influir sobre la decisión de los estudiantes, e incluso de los padres de familia, más aún si se tiene en cuenta que - según la encuesta del DANE (remítase a la Tabla 3 del informe de la Contraloría General de la República) - el desinterés explica el $21 \%$ de las razones por las cuales los niños y los adolescentes abandonan la escuela.

El estudio agrega sobre la función docente y su incidencia en la deserción que, además de las 
obligaciones impuestas por la estandarización y la normatividad de currículos, la actividad de los maestros debe incluir aspectos tales como la formación integral de los estudiantes (preocuparse por el conocimiento, por el ser y los valores que puedan fortalecer y descubrir en ellos), y los incentivos para su permanencia en el sistema (afecto, amistad, confianza, acercamiento a la familia y gestión de condiciones dignas de vida en la escuela), lo que requiere grandes esfuerzos de coordinación, diálogo, trabajo en equipo y ayuda mutua. El informe finaliza indicando cómo otro factor que incide sobre la permanencia escolar se relaciona con la existencia de una pedagogía invisible o de currículos ocultos, con origen en la subjetividad del maestro o de la institución educativa, los cuales inciden a su vez sobre el comportamiento de los estudiantes y afectan sus relaciones con el establecimiento educativo y entre ellos mismos.

\section{Los procesos de evaluación}

Sobre los criterios evaluativos, el documento afirma que otra posible explicación de la deserción escolar es la medida utilizada para determinar el desempeño de los estudiantes. Por una parte, limita la responsabilidad de la educación a aspectos medibles y estandarizables, tales como los cognitivos, memorísticos y técnicos, y deja de lado los complejos factores integrales, sociales y de valores en la formación humana. Es preciso el informe al señalar que la estandarización de los currículos, si bien garantiza unos mínimos exigibles en cuanto a saberes, desplaza o menosprecia la importancia de la diversidad colombiana, los contextos regionales y sociales. Por consiguiente, vuelve trivial la complejidad de una escuela que se construye con la diferencia cultural y étnica, rural y urbana, de modelos y conceptos. Así, la práctica pedagógica, el trabajo colectivo en los procesos institucionales, la construcción de políticas y proyectos pedagógicos integrales, ceden el paso a la preparación forzosa y mecánica para las evaluaciones.

\section{E. Factores exógenos}

Los factores que más inciden en la deserción de los jóvenes del sistema educativo colombiano tienen que ver indiscutiblemente con el entorno social y económico.

\section{Aspectos socio-económicos}

Según el informe de la Contraloría General de la República, tanto en 1997, como en el 2012, la principal razón de inasistencia de los niños en edad escolar (ya sea no asistir o haber asistido y abandonado el centro educativo), es la falta de dinero de su hogar (remítase a la Tabla 3 del informe de la Contraloría General de la República). Otra razón importante es la necesidad de trabajar, la cual solo puede explicarse en un nivel socioeconómico bajo. En la Tabla 3, se presenta una visión general de las variables motivantes de la deserción en este aspecto.

En la Tabla 3, se puede observar cómo las principales causas de retiro de los niños y los jóvenes son, en primer lugar, la falta de dinero, seguido del poco interés, posteriormente la falta de cupos y luego otras causas no determinadas con claridad en la encuesta. Estos resultados ratifican los aspectos que este estudio ha tomado como determinantes en la problemática de la deserción: lo socioeconómico como causa exógena, el interés del individuo hacia la escuela como causa endógena, y la institucionalidad requerida para la cobertura. Estos tres aspectos aparecen en su respectivo orden de porcentaje según la encuesta del DANE.

\section{F. Niveles de pobreza}

Sobre la pobreza, manifiesta el estudio de la Contraloría que, tanto en 1997, como en el 2012, la principal razón de inasistencia de los niños en edad escolar (bien sea no asistir, o bien haber asistido y abandonado el centro educativo) es la falta de dinero de su hogar. Otra razón importante es la necesidad de trabajar, la cual solo puede explicarse en un nivel socioeconómico bajo (remítase a la Tabla 6 del informe de la Contraloría General de la República). Además, con base en la Encuesta Nacional de Demografía y Salud (ENDS), el informe apunta que la deserción escolar femenina en el 2000 se explica en un $56 \%$ por razones económicas. Otra evidencia se encuentra en el crecimiento de la relación entre la tasa global de participación (TGP), y la tasa de deserción, lo cual indica que el problema es creciente, pues cada vez más personas de escasos recursos económicos abandonan sus estudios para ingresar al mercado laboral bajo el fenómeno del trabajador adicional.

\section{G. El desplazamiento forzado}

La vulnerabilidad de los niños desplazados se traduce en la imposibilidad de acceder a cualquier servicio social, no solo por la falta de disponibilidad, sino también por la pérdida de relevancia que esto tiene con respecto a la sobrevivencia misma, antes 
que estudiar o ir al médico es necesario responder al problema del hambre o del sitio donde vivir". ${ }^{17}$ Como indicador del tamaño de este problema, se anota que, según el Registro Nacional de Población desplazada por la Violencia de la Presidencia de la República, el número acumulado de población desplazada entre 5 y 17 años es de 278000 personas a septiembre del 2012. En el caso específico de la población desplazada hacia Bogotá, se observa que alrededor del $60 \%$ corresponde a personas en edad escolar, de los cuales el $73 \%$ no está vinculado a ninguna institución educativa.

\section{Deserción en la Institución Siete de Agosto}

Para la aproximación al fenómeno de la deserción en la Institución Siete de Agosto, se asumió como criterio de análisis el retiro de niños y jóvenes de la institución en el periodo 2005- 2008, a fin de explorar y describirlas causas de dicho retiro.

Los referentes estadísticos muestran una cifra de cobertura en el periodo 2005- 2008 de 1806 estudiantes, distribuidos en los diferentes niveles educativos que ofrece la institución. Para el periodo escolar siguiente (2006-2007), la institución cuenta con 1955 estudiantes, lo que significó un $8,25 \%$ de aumento en la población atendida. El informe del proceso de matrícula para el periodo 2007-2008 registra una cifra de 1494 estudiantes.

Es claro que se observa una disminución relevante en el número de matriculados en el último periodo, es decir, 461 estudiantes retirados, lo que equivale a un 23,53 \% en el índice de deserción para la institución. Esta situación sobrepasa ampliamente las tasas de deserción a nivel municipal que, según datos de la Secretaría de Educación Municipal, es del $4 \%$. Igualmente, supera el índice de deserción departamental que se encuentra en un $3 \%$. Es conveniente señalar que las cifras locales y regionales están por debajo de la nacional, la cual para el 2007 se presentó en un $5,60 \%$.

Estas preocupantes cifras de retiro de niños y jóvenes de la Institución Educativa Siete de Agosto se convierten en un interrogante que bien vale la pena develar. Para ello se determinó iniciar un proceso de búsqueda de esta población, a fin de indagar las razones por las cuales habían abandonado la institución.

17. Véase Contraloría General de la República-Dirección de Estudios Sectoriales, supra, nota 3.
Esta es una labor muy exigente, ya que los desertores son personas que no mantienen contacto actual con la institución.

\section{A. La palabra de los desertores}

El acercamiento a los desertores se realizó mediante una encuesta directa, por medio de una llamada telefónica en la cual se indagaba en torno a las razones por las que había dejado la Institución Siete de Agosto. Se logró ubicar a 217; de ellos, el mayor motivo de deserción de los niños y jóvenes es el cambio de domicilio con un $60,83 \%$, hecho que parece estar asociado a las problemáticas de desplazamiento y movilidad intraurbana.

El desplazamiento intraurbano, como variable de deserción confirmada en la Institución Educativa Siete de Agosto, es una situación compleja, pero la escuela no puede ser ajena a estas problemáticas de tipo social y económico que aumentan los niveles de deserción escolar y cortan abruptamente los procesos formativos de los estudiantes.

Este fenómeno aparece articulado al actual proceso de transformación en la conformación poblacional de la ciudad. La Cali de hoy la conforman colonias de diferentes lugares del país, una gran población desplazada por diversas razones y que se estima en 53500 personas que integran 10700 hogares, en su mayoría mujeres y menores de edad, provenientes de regiones agrarias o expulsadas de zonas urbanas de la misma ciudad..$^{18}$

Por otra parte, se encuentra el fenómeno de violencia generalizada de las últimas décadas, el cual se convierte entonces en un factor fundamental en la movilidad interurbana, llegando incluso a ser aceptado por la Corte Constitucional Colombiana como desplazamiento intraurbano. ${ }^{19}$

La encuesta ofrece otro panorama de respuestas interesantes, como es el retiro de los estudiantes por la convivencia en la institución, con una tasa del 8,76\%. Respuestas como "por la distancia", con un 5,53\%; "por reprobación", con un 3,23\%; "no hay énfasis", con un 1,38 \%; y por "el nivel académico", como respuesta motivante de los retiros con un $0,92 \%$, entre otras, muestran una amplia diversidad en cuanto a las razones para dejar la institución. Sin embargo, cada

18. Proyección realizada a partir de los datos de la conferencia episcopal (1985-1994), y de la consultoría para los derechos humanos y el desplazamiento coDHes (1995-96).

19. Véase Corte Constitucional Colombiana. Sentencia T-025/ 04. (MP Manuel Cepeda Espinosa: Enero 22 del 2004). 
una de ellas es valiosa cuando se pretende encontrar motivos y soluciones al problema de la deserción.

\section{B. Una mirada hacia el interior de la institución}

Tal como aparecen las cosas, es pertinente reflexionar sobre lo que acontece al interior de la escuela, ya que existen factores endógenos que motivan la deserción, asunto que amerita un análisis cuidadoso en cuanto a la calidad y satisfacción en la prestación del servicio, puesto que los estudiantes que actualmente se encuentran en la institución se convierten en desertores potenciales.

Para realizar esta mirada a los procesos internos de la Institución Educativa Siete de Agosto, se utilizó la encuesta de satisfacción elaborada con la participación de padres de familia, estudiantes y docentes. En ella se indaga sobre el desarrollo de los procesos en las áreas de gestión académica, social y administrativa.

En el análisis general de la encuesta de satisfacción, la gestión académica se destaca como indicador; la respuesta de totalmente satisfecho y satisfecho alcanzó un porcentaje final con relación al número de respuestas del $36,13 \%$, seguido por la gestion social con un $27,17 \%$. La gestion administrativa supera a las demás áreas de gestión en la respuesta medianamente satisfecho e insatisfecho, lo cual muestra esta área urgida de estrategias de mejoramiento para la optimización de los recursos y la satisfacción de los usuarios del servico educativo que se presta; al final señaló un porcentaje del 18,42\%.

En el área de gestión social, se debe señalar, tambien a manera de conclusión, que los padres de familia, estudiantes y docentes coinciden en un considerable grado de insatisfacción en el criterio "ambiente escolar", siendo este factor consecuente con uno de los motivos expresados por los desertores.

La gestión administrativa mostró, en términos generales, un repunte de mediana y ninguna satisfacción en el desarrollo de sus procesos con relación a las otras áreas de gestión. La planta física, el mejoramiento de la misma y la atención de quejas, soluciones y reclamos, se muestran como los criterios de mayor inconformidad en los padres de familia y los estudiantes.

\section{UNAS PROPUESTAS}

\section{HACIA LA RETENCIÓN}

La identificación de factores exógenos y endógenos determinantes de la deserción implica que, desde la escuela, se piensen y diseñen estrategias tendientes a fortalecer el deseo de los niños y jóvenes por permanecer en las instituciones educativas, con el propósito de que sean espacios atractivos en donde, en determinada instancia, sean tenidos en cuenta al momento de tomar decisiones.

En este sentido, la escuela debe pensar su compromiso con la comunidad, posibilitar la participación activa y solidaria de los padres de familia y los representantes del sector productivo. La consolidación de las asociaciones de padres y el fortalecimiento de los lazos de cooperación con las entidades locales, aparecen como herramientas claves en el alcance de este propósito. La escuela ha de tener, entre sus factores de éxito, la retención de los niños y jóvenes, por lo tanto, la implementación de proyectos educativos alternativos debe generar propuestas claras encaminadas al ofrecimiento de opciones atractivas que respondan a las necesidades de estudiantes.

La gestión administrativa es una variable identificada como fundamental, si lo que se busca es pensar en retener a los estudiantes en las instituciones. Por esto, es necesario preguntarse por la calidad de la educación que se presta a la comunidad, de modo que las vías de comunicación son claves para que padres de familia y estudiantes puedan manifestar sus percepciones. Las encuestas no son suficientes, se requiere de mecanismos reales de escucha y participación.

En este orden, es importante señalar que corresponde a la función administrativa, dentro de una concepción gerencial de las instituciones educativas, generar las estrategias y los mecanismos que permitan dinamizar los ambientes escolares, la dignidad de vida y la continuidad de los jóvenes en las instituciones; una cultura organizacional y gerencial puede ayudar a disminuir los niveles de deserción escolar.

Por último, y teniendo en cuenta la priorización de las situaciones problemáticas que movilizan el retiro de los jóvenes de las instituciones escolares, se requiere atender los problemas de convivencia y el ambiente escolar, así como entender sus relaciones con las diversas violencias que predominan en las ciudades colombianas. La complejidad de interrelaciones que se suscitan al interior de la escuela está en relación directa con el entorno cercano de los jóvenes y de la escuela misma.

\section{CONCLUSIONES}

Al finalizar el estudio exploratorio sobre la deserción escolar en la Institución Educativa Siete de Agosto, se llega a las siguientes conclusiones: 
- El nivel de deserción escolar en la institución está determinado por factores externos e internos.

- El factor externo determinante de la deserción escolar — según el concepto de los niños y los jóvenes desertores- es el cambio de domicilio, lo que demuestra que existe una problemática de movilidad y desplazamiento intraurbano en la comunidad educativa objeto del estudio realizado.

- El estudio realizado sobre el desarrollo de los procesos internos en la institución muestra, en términos generales, satisfacción en las áreas de gestión académica y social. El área de gestión administrativa no presenta un nivel de satisfacción entre la comunidad educativa, lo cual debe ser tenido en cuenta debido a que las personas encuestadas, especialmente los padres y los estudiantes, se convierten en potenciales desertores.

- La convivencia y la disciplina se muestran como variables internas de la institución al momento de tomar decisiones sobre la continuidad de los estudios. Los desertores entrevistados vía telefónica sobre el motivo por el cual se retiraron, y los miembros de la comunidad educativa indagados en la encuesta de satisfacción, coinciden en este concepto.

- Los ejercicios investigativos al interior de la institución conducen a identificar problemáticas y soluciones pertinentes con la realidad de la escuela y su entorno.

- Se requieren propuestas innovadoras y atractivas desde y hacia la escuela, con el objetivo de retener a los jóvenes dentro del sistema educativo.

- Las estrategias propuestas se pueden tomar como plataforma para futuras investigaciones en ese contexto.

- Los aspectos misionarios y visionarios de la institución objeto de estudio requieren de una revisión a partir de los resultados obtenidos en esta investigación.

\section{REFERENCIAS}

Acto legislativo 01 del 2001.Por medio del cual se modifican algunos artículos de la Constitución Política. Agosto 1 del 2001. DO44506.

Comisión Económica para América Latina y el Caribe-CEpal. Deserción Escolar en América latina. InFORME. CEPAL.(2002).

Contraloría General de La República-Dirección de Estudios Sectoriales. LA DESERCIÓN ESCOLAR EN LA EDUCACIÓN BÁSICA Y MEDIA. (2004)

Constitución Política de Colombia [Const]. Título II de los Derechos, garantías y deberes, Capítulo I de los Derechos Fundamentales. Julio 7 de 1991 (Colombia).

Corte Constitucional Colombiana. Sentencia T-025/04. (MP Manuel Cepeda Espinosa: Enero 22 del 2004).

Eduardo Aldana-Valdés et al. Misión, Ciencia, Educación y Desarrollo. Tomo 1. Informe de LA Misión de lOS SABios. Colombia: AL FILO DE LA OPORTUNIDAD. Colciencias, TM Editores.

Ley 115 de 1994. Por la cual se expide la ley general de educación. Febrero 8 de 1994. Disponible en http://www. mineducacion.gov.co/1621/articles-85906_archivo_ pdf.pdf

Ley 715 del 2001.Por la cual se dictan normas orgánicas en materia de recursos y competencias de conformidad con los artículos 151, 288, 356 y 357 (Acto Legislativo 01 de 2001) de la Constitución Política y se dictan otras disposiciones para organizar la prestación de los servicios de educación y salud, entre otros. Disponible en http://www.mineducacion.gov.co/1621/articles-86098_archivo_pdf.pdf

Ministerio de Educación Nacional. Colombia reforma CURRICULAR. (1978).

Ministerio de Educación Nacional. Sistema Nacional DE Indicadores Educativos para los Niveles de Preescolar, Básica y Media en Colombia. Disponible en: https://www.mineducacion.gov.co/1621/articles-329021_archivo_pdf_indicadores_educacion.pdf

Vincent Tinto. Definir la deserción: una cuestión de perspectiva. Revista de Educación Superior 71. 1989. Pág. 1-43. 